\title{
Intravenous acetaminophen for postoperative supratentorial craniotomy pain: a prospective, randomized, double-blinded, placebo-controlled trial
}

\author{
Walavan Sivakumar, MD, ${ }^{1}$ Michael Jensen, BS, Julie Martinez, MSN, RN, ${ }^{3}$ Michael Tanana, PhD, ${ }^{4}$ \\ Nancy Duncan, MSN, FNP, ${ }^{1}$ Robert Hoesch, MD, PhD, ${ }^{2,3}$ Jay K. Riva-Cambrin, MD, ${ }^{1,5}$ \\ Craig Kilburg, MD, ${ }^{1}$ Safdar Ansari, MD, ${ }^{2}$ and Paul A. House, MD'
}

\begin{abstract}
Departments of ${ }^{1}$ Neurosurgery and ${ }^{2}$ Neurology, Clinical Neurosciences Center, University of Utah, Salt Lake City; ${ }^{3}$ Neurosciences Clinical Program, Intermountain Healthcare, Murray; ${ }^{4}$ Department of Biostatistics, Biosocial Research Institute, University of Utah, Salt Lake City, Utah; and ${ }^{5}$ Department of Clinical Neurosciences, University of Calgary, Alberta, Canada
\end{abstract}

\begin{abstract}
OBJECTIVE Acute pain control after cranial surgery is challenging. Prior research has shown that patients experience inadequate pain control post-craniotomy. The use of oral medications is sometimes delayed because of postoperative nausea, and the use of narcotics can impair the evaluation of brain function and thus are used judiciously. Few nonnarcotic intravenous (IV) analgesics exist. The authors present the results of the first prospective study evaluating the use of IV acetaminophen in patients after elective craniotomy.
\end{abstract}

METHODS The authors conducted a randomized, double-blinded, placebo-controlled investigation. Adults undergoing elective, supratentorial craniotomies between September 2013 and June 2015 were randomized into two groups. The experimental group received $1000 \mathrm{mg} / 100 \mathrm{ml}$ IV acetaminophen every 8 hours for 48 hours. The placebo group received $100 \mathrm{ml}$ of $0.9 \%$ normal saline on the same schedule. Both groups were also treated with a standardized pain control algorithm. The study was powered to detect a $30 \%$ difference in the primary outcome measures: narcotic consumption (morphine equivalents, ME) at 24 and 48 hours after surgery. Patient-reported pain scores immediately postoperatively and 48 hours after surgery were also recorded.

RESULTS A total of 204 patients completed the trial. No significant differences were found in narcotic consumption between groups at either time point (in the treatment and placebo groups, respectively, at 24 hours: $84.3 \mathrm{ME}[95 \% \mathrm{Cl}$ 70.2-98.4] and 85.5 ME [95\% Cl 73-97.9]; and at 48 hours: $123.5 \mathrm{ME}$ [95\% $\mathrm{Cl} 102.9-144.2]$ and $134.2 \mathrm{ME}[95 \% \mathrm{Cl}$ 112.1-156.3]). The difference in improvement in patient-reported pain scores between the treatment and placebo groups was significant $(p<0.001)$.

CONCLUSIONS Patients who received postoperative IV acetaminophen after craniotomy did not have significantly decreased narcotic consumption but did experience significantly lower pain scores after surgery. The drug was well tolerated and safe in this patient population.

Clinical trial registration no.: NCT01948505 (clinicaltrials.gov)

https://thejns.org/doi/abs/10.3171/2017.10.JNS171464

KEYWORDS neurosurgery; craniotomy; pain; acetaminophen; narcotic; analgesia

$\mathrm{P}$ ROVIDING acute postoperative pain control after cranial surgery remains a clinical challenge. Numerous studies have shown that patients in the neurocritical care (NCC) unit experience inadequate pain control. . $^{3,4,6,9,13}$ Optimizing patient comfort after craniotomy is often dif- ficult because the use of narcotic medications can impair the clinical evaluation of neurological function. The sedation and pupillary miosis caused by opioids can directly mask seminal signs of intracranial pathology, and thus, opioids are used judiciously. Additionally, craniotomy is

ABBREVIATIONS IV = intravenous; NCC = neurocritical care; VAS = visual analog scale.

SUBMITTED June 16, 2017. ACCEPTED October 30, 2017.

INCLUDE WHEN CITING Published online April 20, 2018; DOI: 10.3171/2017.10.JNS171464. 
associated with postoperative nausea, which delays the use of oral medications. Very few nonnarcotic intravenous (IV) medications are available to this patient population.

The recently approved IV formulation of acetaminophen (Ofirmev injection, Cadence Pharmaceuticals) might serve as a safe, potent, nonopioid adjunct to narcotic medicine that may improve patient comfort and care. Multiple studies have shown that, compared with placebo, IV acetaminophen significantly decreases opioid requirements in patients postoperatively. ${ }^{7,12,17}$ Although this decreased opioid requirement could be of special importance in patients postcraniotomy, the drug has yet to be tested in a controlled fashion within the neurosurgical population. After recently beginning to use the drug selectively and identifying anecdotal benefits in regard to pain control, lack of sedation, and early ambulation, we designed this trial to compare IV acetaminophen as a scheduled adjunct with our existing standardized craniotomy pain control regimen. Additionally, given the recent significant increase in the cost of the medication in a time of interest in health care cost containment, this clinical investigation was especially timely. We hypothesized that patients receiving IV acetaminophen, starting intraoperatively, would experience less pain and that this pain reduction would lead to decreased narcotic requirements in the first 48 hours after surgery.

\section{Methods}

\section{Study Design and Oversight}

We conducted a prospective, single-center, randomized, double-blinded, placebo-controlled study. The study protocol was approved by the university institutional review board and carried out in compliance with the requirements of the Health Insurance Portability and Accountability Act (HIPAA). An investigational new drug exemption was acquired from the US FDA prior to study initiation. The study was registered with the National Institutes of Health at clinicaltrials.gov (registration no. NCT01948505). All authors assume responsibility for the accuracy and completeness of the data and analyses.

\section{Study Population and Enrollment}

We recruited patients undergoing elective supratentorial craniotomies at the University of Utah. Reasons for craniotomies included tumor resection and biopsies, aneurysm clipping, resection of vascular malformations, and epilepsy surgery. Consent to participate in the study was acquired preoperatively from the patient directly or from a legally authorized representative prior to administration of the first dose. Included patients were older than 18 years, weighed more than $50 \mathrm{~kg}$, and were admitted to the NCC unit at the completion of the surgical procedure. Patients were excluded if they had a posterior fossa craniotomy, a documented allergy to acetaminophen, or severe hepatic or renal impairment; were pregnant or breastfeeding; or were unable to provide consent and lacked a physically present legally authorized representative.

\section{Study Treatment}

Patients were randomly assigned to receive $1000 \mathrm{mg}$ of
IV acetaminophen in a $100-\mathrm{ml}$ saline solution or $100 \mathrm{ml}$ of $0.9 \%$ normal saline every 8 hours for 48 hours. The first dose of the study medication was given intraoperatively at the completion of the procedure.

\section{Randomization}

Randomization was performed with the use of a blockof-4 randomization scheme. Randomization was performed by the investigational drug service at the university hospital, thereby maintaining the double-blind aspect of the study (patients and study personnel/investigators). Patient study identification numbers were recorded in an Excel database, randomized permuted blocks of 4 were created, and patients were allotted to either the acetaminophen or placebo group by the pharmacy.

\section{Study Flow}

Upon acquisition of patient consent, the pharmacy was notified, and the study drug (or placebo) was transported to the operating room to be given at the conclusion of the operative case. The timing of study drug administrations was coordinated among the pharmacy, the anesthesiologists, and the NCC nurses.

All patients received a standardized NCC unit postoperative craniotomy pain protocol (including the narcotics fentanyl, morphine, hydromorphone, and oxycodone) in addition to the study drug or placebo. During the course of the trial, a strict moratorium was placed on ordering acetaminophen-containing combination drugs such as Lortab and Percocet as well as on ordering additional orally administered acetaminophen to avoid exceeding the recommended daily limit of acetaminophen of $4 \mathrm{~g}$.

\section{Outcomes}

The primary efficacy end point was narcotic consumption during the first 48 hours after craniotomy. Opioid usage was calculated by converting the amount of the narcotics taken by the patient into oral morphine equivalents (ME), which were totaled for each postoperative day. During the postoperative period, patients had ready access to the aforementioned narcotic medications. As per NCC unit protocol, narcotic consumption and pain scores are reported every 4 hours. If the patient requests more pain medication, the narcotic dose is increased or the narcotic used is changed. The patient is then assessed and treated hourly with these interventions until adequate pain relief is achieved. Data on narcotic usage and pain scores were collected prospectively through postoperative day 2, or 48 hours after the initial administration of the study drug.

Pain scores were collected prospectively during the 48hour postoperative period using the visual analog scale (VAS). ${ }^{1}$ Patients were asked to rate their pain on a $0-10$ scale, where " 0 " represented "no pain" and " 10 " represented the "worst pain I've ever experienced." Data were recorded by the nursing staff every 4 hours or hourly if additional pain medication was required.

Other secondary information recorded by study personnel included narcotic-related side effects like nausea, vomiting, urinary retention as indicated by replacement of the Foley catheter, and constipation requiring escalation 


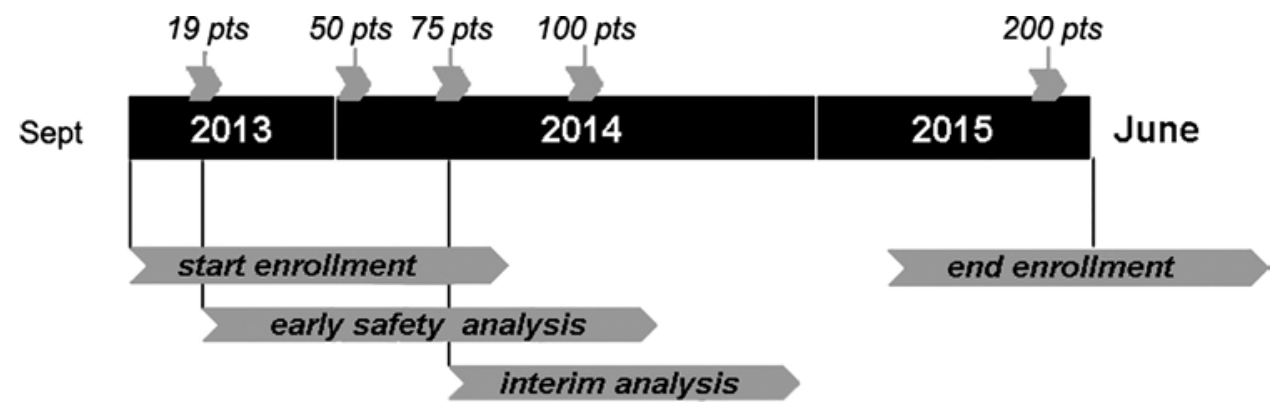

FIG. 1. IV acetaminophen trial timeline. pts = patients.

of the NCC unit bowel care regimen. Time to first and maximal ambulation, NCC unit length of stay, and overall hospital length of stay were recorded. The number of CT scans and MR images ordered during the study period was also recorded.

The principal adverse-event data recorded included the incidence of clinically relevant transaminitis and new documentation of acetaminophen allergy. All patients underwent review performed by the NCC unit team twice daily, and all potential acetaminophen-related adverse effects were reported to the study team. A hepatic panel (including alkaline phosphatase, aspartate aminotransferase, alanine aminotransferase, and total and direct bilirubin) was assessed for any patient with new complaints of significant abdominal pain, weakness, or jaundice. Any new evidence of transaminitis in a patient with any of the aforementioned symptoms would result in immediate cessation of the study drug.

\section{Statistical Analysis}

A power analysis indicated that 210 patients $(105$ per group) would be required to see a $30 \%$ reduction in narcotic requirement (using a significance level of 5\% and assuming gaussian distribution with a power level of 80\%). Continuous baseline characteristics were compared using 2-tailed independent t-tests, and chi-square tests were used for analyzing categorical data. All mean values are expressed with $95 \%$ confidence intervals. Statistical significance was defined as $p<0.05$. The analyses were performed using SPSS for Windows (SPSS Inc.).

\section{Results}

\section{Enrollment}

The enrollment goal was reached in a period of 85 weeks spanning between September 2013 and June 2015. Figure 1 shows the trial timeline and illustrates a steady enrollment process. An early safety analysis was performed at 1 month. An interim analysis was performed after 6 months, the results of which were presented at the 82nd AANS Annual Scientific Meeting..$^{15}$ No adjustments were made as a result of these early analyses.

\section{Patient Population}

Figure 2 shows the enrollment and randomization for the trial. Overall, 243 patients were screened and identified as eligible. Thirty-one patients were not included in randomization: 15 eligible patients declined to participate and 16 patients were not randomized because of the logistical limitations of the enrollment process (e.g., lack of a study coordinator to obtain patient consent). Among the 212 patients who were randomized, 106 were allocated to

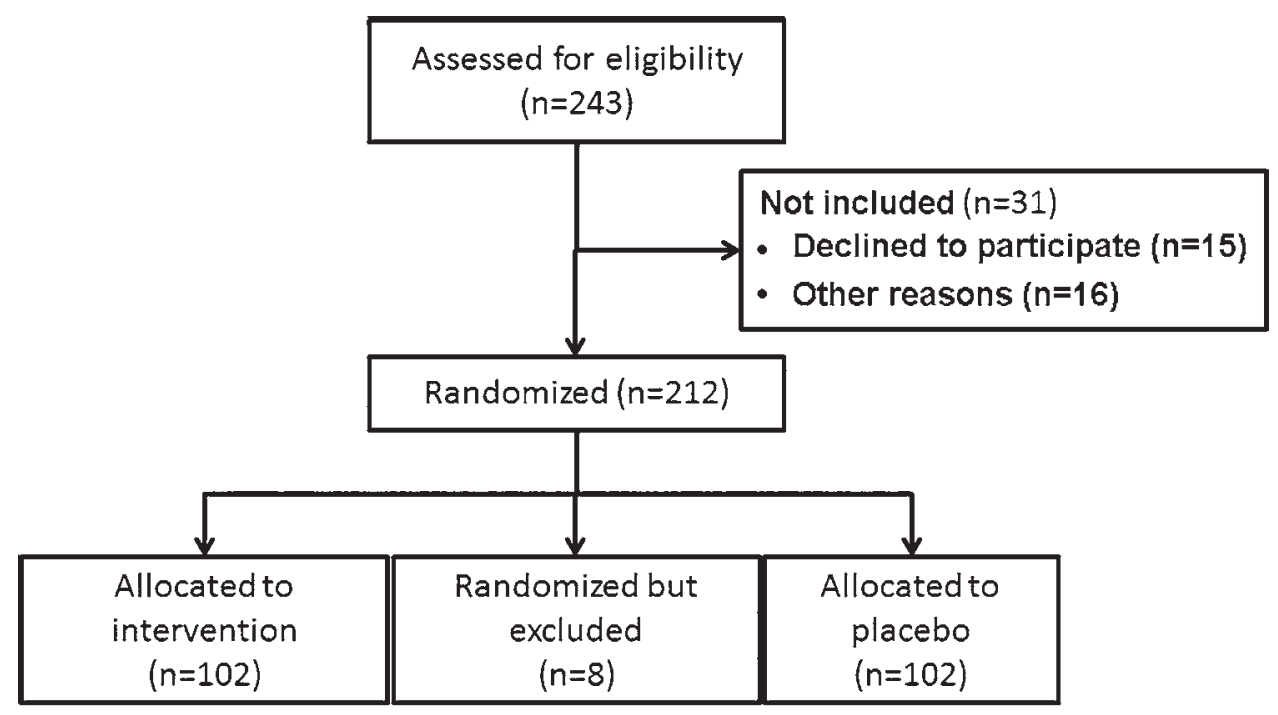

FIG. 2. CONSORT flow diagram of trial enrollment. 
TABLE 1. Preoperative patient characteristics

\begin{tabular}{|c|c|c|c|}
\hline Variable & $\begin{array}{l}\text { Placebo } \\
(n=102)\end{array}$ & $\begin{array}{l}\text { Treatment } \\
(n=102)\end{array}$ & $\begin{array}{c}\mathrm{p} \\
\text { Value }\end{array}$ \\
\hline Mean age in yrs $(95 \% \mathrm{Cl})$ & $50.3(47.4-53.2)$ & $50.6(47.6-53.5)$ & 0.89 \\
\hline Male sex, $\mathrm{n}(\%)$ & $48(47.1)$ & $31(30.4)$ & 0.02 \\
\hline $\begin{array}{l}\text { Mean admission wt in } \mathrm{kg} \\
\quad(95 \% \mathrm{Cl})\end{array}$ & $88.2(83.7-92.8)$ & $80.3(76.7-83.8)$ & 0.01 \\
\hline Ethnicity, n (\%) & & & 0.69 \\
\hline Hispanic or Latino & $8(7.8)$ & $5(4.9)$ & \\
\hline Not Hispanic or Latino & $91(89.2)$ & $94(92.2)$ & \\
\hline Unknown/not reported & $3(2.9)$ & $3(2.9)$ & \\
\hline Race, n (\%) & & & 0.16 \\
\hline $\begin{array}{l}\text { American Indian/ } \\
\text { Alaska Native }\end{array}$ & $1(1.0)$ & $4(3.9)$ & \\
\hline Asian & $3(2.9)$ & 0 & \\
\hline $\begin{array}{l}\text { Native Hawaiian/Pa- } \\
\text { cific Islander }\end{array}$ & $2(2.0)$ & $1(1.0)$ & \\
\hline African American & 0 & $1(1.0)$ & \\
\hline White & $86(84.3)$ & $91(89.2)$ & \\
\hline Unknown/not reported & $10(9.8)$ & $5(4.9)$ & \\
\hline
\end{tabular}

the placebo group and 106 were allocated to the intervention group. Eight of these patients (4 in each group) were initially randomized but were removed from the study and analysis because of untimely administration of the study drug or transfer of the patient to a unit other than the NCC unit postoperatively. These patients did not receive subsequent doses of the study drug, as they would be inadequately monitored and therefore were not included in evaluation of the primary and secondary outcomes.

Baseline characteristics of patients in the intervention and placebo groups are shown in Table 1. Patients in the two groups were comparable with regard to age and ethnicity. There were more males in the placebo group than in the treatment group (48 [47.1\%] vs 31 [30.4\%], $\mathrm{p}=0.02)$. There is not a known sex-specific variance in acetaminophen efficacy. Additionally, the mean patient weight was heavier in the placebo group ( $88.2 \mathrm{~kg}$ vs $80.3 \mathrm{~kg}, \mathrm{p}=0.01$ ).

\section{Primary Outcome Measure}

At 48 hours after surgery, patients who received IV acetaminophen in addition to our standardized pain protocol did not have a statistically significantly lower mean narcotic requirement, measured in ME, when compared with those in the placebo group (123.5 ME [95\% CI 102.9-144.2] vs 134.2 ME [95\% CI 112.1-156.3], $\mathrm{p}=0.49$; Table 2). There was a trend toward lower narcotic requirement during the 2 nd postoperative day for patients in the treatment group (39.2 ME [95\% CI 30.2-47.7] vs $48.7 \mathrm{ME}$ [95\% CI 37.5-60]); however, this difference was not statistically significant $(\mathrm{p}=0.18)$.

\section{Patient-Reported Pain Scores}

The difference in improvement in patient-reported pain scores between the treatment and placebo groups was significant ( $<<0.001$; Fig. 3). Although the mean patient-
TABLE 2. Narcotic requirements in morphine equivalents

\begin{tabular}{rccc}
\hline Time Point & Placebo $(95 \% \mathrm{Cl})$ & Treatment $(95 \% \mathrm{Cl})$ & p Value \\
\hline 0-24 hrs & $85.5(73-97.9)$ & $84.3(70.2-98.4)$ & 0.90 \\
\hline 24-48 hrs & $48.7(37.5-60)$ & $39.2(30.7-47.7)$ & 0.18 \\
\hline $0-48 \mathrm{hrs}$ & $134.2(112.1-156.3)$ & $123.5(102.9-144.2)$ & 0.49 \\
\hline
\end{tabular}

reported pain scores began at the same level in the treatment and placebo groups, the treatment group experienced a more rapid decrease in pain score and ultimately lower pain scores than the placebo group. At 24 hours after surgery, these differences were noted to be significant $(\mathrm{p}<$ 0.001 ). This difference did not retain its significance at 48 hours.

\section{Secondary In-Patient Outcome Measures}

There were no statistically significant differences in mean hospital length of stay between the placebo and treatment groups (5.7 vs 5.6 days, $p=0.94$ ), and there were no differences in the mean rates of Foley catheter removal $(91.2 \%$ vs $95.1 \%, \mathrm{p}=0.27)$, suppository use $(0.5 \%$ vs $0.6 \%$, $\mathrm{p}=0.11)$, or enema use $(0 \%$ vs $0 \%, \mathrm{p}=0.15)$ within the first 72 hours (Table 3 ). In regard to postoperative patient activity, there were no significant differences between maximum ambulation (347.7 feet vs 326.4 feet, $p=0.59$ ), mean number of stairs climbed (3.2 vs $1.8, \mathrm{p}=0.38$ ), or rates of discharge from physical therapy within 48 hours $(19.6 \%$ vs $15.8 \%, p=0.73)$. There were also no significant differences in the mean utilization of postoperative CT $(0.6 \%$ vs $0.5 \%, \mathrm{p}=0.49)$ or $\operatorname{MR}(0.4 \%$ vs $0.5 \%, \mathrm{p}=0.63)$ imaging.

\section{Safety}

No adverse events, including death, liver enzyme elevation, and allergic reaction, attributable to the study medication were noted in this study.

\section{Discussion}

Intravenous opioids have long been the primary source of perioperative analgesia for patients experiencing moderate to severe pain. The judicious use of opioids (to avoid side effects and depression of neurological function during examination) has resulted in chronic undertreatment of pain in patients who have undergone elective intracranial surgery. Recently, there has been an increased interest in adding nonopioid analgesics to postoperative pain algorithms. ${ }^{16}$ Given the common occurrence of nausea precipitated by cranial surgery and opioids and the subsequent delayed oral intake, there is a need for intravenously administered agents. This study was an investigator-initiated, single-center, prospective, randomized, doubleblinded, placebo-controlled trial aimed at assessing the effects of IV acetaminophen on narcotic utilization and patient perception of pain in the postoperative period after craniotomy. Our investigation is the first prospective study to evaluate these effects.

We hypothesized that patients receiving IV acetaminophen, starting intraoperatively, would experience less pain and that this pain reduction would lead to decreased nar- 


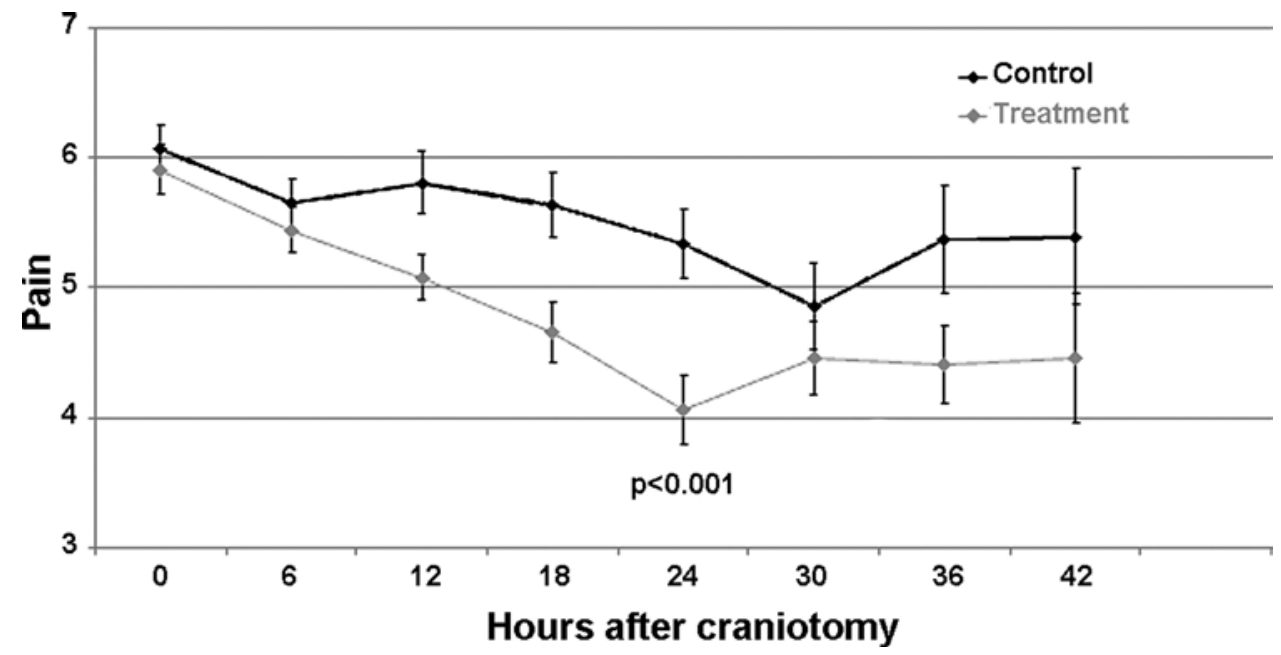

FIG. 3. Patient-reported pain scores over time. At 24 hours, the difference in patient-reported pain scores between the two groups was significant $(p<0.001)$. The difference was no longer significant at 48 hours.

cotic requirements in the first 48 hours after surgery. The study was powered to detect a $30 \%$ difference in narcotic usage, as this was thought to represent a clinically meaningful difference. We found, however, only a modest reduction in total narcotic usage, and the primary end point was not met.

Because we previously noted (anecdotally) perceived benefits in pain control, nausea, and early ambulation with the use of IV acetaminophen, we prospectively tracked several secondary factors identified a priori to explore possible benefits. Of these secondary factors, there was significant improvement in patient-perceived postoperative pain control. There was no significant difference, however, between the group that received intraoperative acetaminophen and the group that did not in many important factors, such as episodes of emesis, time to ambulation, and overall length of stay.

The observed reduction in postoperative craniotomy pain contradicted the lack of reduction in narcotic use and narcotic-associated complications. There are several probable explanations for our findings. First, IV acetaminophen may provide only a modest improvement in pain control. While the addition of the drug may be clinically helpful, the improvement in pain control is not sufficient to allow for significant narcotic reduction. Second, the utilization of a standardized pain protocol in both study groups might not allow the maximum potential narcotic reduction to be revealed. Further investigations could prospectively limit narcotic use to evaluate the potential of acetaminophen substitution. We did not employ this study design, as we lacked a priori estimates of the true efficacy of acetaminophen. Third, our study was not powered to demonstrate modest $(<30 \%)$ reduction in narcotic usage in a statistically significant manner. A small reduction in total narcotic use was noted; however, a 30\% cutoff was chosen as a clinically meaningful change, and this primary outcome was not reached. Another trial with a larger sample size might show a significant reduction in narcotic use, either above or below the $30 \%$ cutoff.

Narcotics and acetaminophen are known to reduce pain through different pathways. Although the actions of acetaminophen at the molecular level have not been clearly

TABLE 3. Secondary outcome measures

\begin{tabular}{lccc}
\hline \multicolumn{1}{c}{ Variable } & Placebo & Treatment & p Value \\
\hline Hospital length of stay in days & $5.7(4.9-6.4)$ & $5.6(4.8-6.5)$ & 0.94 \\
\hline No. of antiemetic doses w/in 72 hrs & 3.7 & 4.5 & NS \\
\hline$\%$ w/ Foley catheter removed w/in 72 hrs & 91.2 & 95.1 & 0.27 \\
\hline$\%$ w/ bowel movement in 1st 72 hrs & 65.3 & 64.7 & 0.01 \\
\hline Suppositories in 1st 72 hrs & $0.5(0.3-0.6)$ & $0.6(0.5-0.8)$ & 0.11 \\
\hline Enema w/in 72 hrs & $0(0)$ & $0(0-0.1)$ & 0.15 \\
\hline Maximum ambulation in feet & $347.7(289-406)$ & $326.4(274.1-378.8)$ & 0.59 \\
\hline Stairs climbed (95\% Cl) & $3.2(1.6-4.8)$ & $1.8(0.9-2.7)$ & 0.38 \\
\hline$\%$ w/ PT discharge w/in 48 hrs & 19.6 & 15.8 & 0.73 \\
\hline CT scans w/in 48 hrs & $0.6(0.4-0.7)$ & $0.5(0.4-0.7)$ & 0.49 \\
\hline MRl scans w/in 48 hrs & $0.4(0.3-0.5)$ & $0.5(0.4-0.6)$ & 0.63 \\
\hline
\end{tabular}

NS = not significant; $\mathrm{PT}=$ physical therapy.

Values are means $(95 \% \mathrm{Cl})$ unless stated otherwise. 
elucidated, the drug is thought to work partly as a weak inhibitor of central prostaglandin synthesis. ${ }^{2}$ On the other hand, narcotics work on opiate receptors, causing a reduction in neuronal excitability at levels including the dorsal spinal horn and also the brainstem. ${ }^{11}$ The failure of the current study to achieve the primary end point, despite a positive result in the secondary end point, might be partially due to these differing mechanisms. One could theorize, for example, that while acetaminophen reduces pain, narcoticinduced limbic desensitization is also needed to reduce anxiety associated with craniotomy. The primary hypothesis of the current study was not based on known mechanisms of action, however, but rather on extant data revealing that the use of intravenous acetaminophen has reduced overall opioid use in other surgical procedures. ${ }^{7,12,17}$

Our results correlate with the findings of a retrospective analysis by Hoefnagel et al., ${ }^{5}$ which failed to show a benefit in opioid consumption in postcraniotomy patients who received a single dose of intraoperative IV acetaminophen. Interestingly, the cost of IV acetaminophen increased $170 \%$ from the start of our trial $(\$ 12.90$ per $1-\mathrm{g}$ vial in $2013, \$ 35.05$ per $1-\mathrm{g}$ vial by the end of the trial). Although the costs of IV acetaminophen are still relatively low, our results raise the question of whether the relatively moderate benefit in pain achieved by most patients is worth the cost.

\section{Other Nonnarcotic Adjuncts}

Other studies have demonstrated modest, if not clinically significant, benefits in patient-related outcomes after craniotomies using other nonnarcotic adjuncts. Molnár et al. ${ }^{8}$ found a modest decrease (1-2 points on the VAS) in postoperative pain scores (at evening, postoperative day 1 , and postoperative day 5) in patients receiving a single dose of preoperative diclofenac. Because none of their patients required postoperative opioid analgesia and their sample population included both supra- and infratentorial craniotomies, it is difficult to compare the two patient populations. Peng et al. ${ }^{10}$ found a modest, immediate improvement in postoperative pain scores and tramadol consumption 30 minutes and 2 hours after surgery following intraoperative dexmedetomidine use, but no such difference remained at 24 hours. Shimony et al. ${ }^{14}$ also showed a modest early improvement in pain scores at 24 hours when using perioperative pregabalin (numerical rating scale score $1.5 \mathrm{vs}$ $2.3, \mathrm{p}<0.01$ ), but this difference did not continue on postoperative days 2 and 3 . Moreover, their patient population consisted largely of patients who underwent infratentorial craniotomies, limiting the generalizability of this data. When taken as a whole, the data to date do not reveal a clear best nonnarcotic agent for pain reduction.

At our institution, these results have not led to adoption of IV acetaminophen use for all craniotomy patients, as most clinically important parameters were not improved. However, for patients with prolonged nausea or narcotic sensitivity, IV acetaminophen is routinely employed. Additionally, the medication is frequently used when pain control is not rapidly achieved using standard protocols.

\section{Limitations}

This study has some limitations. First, this study popu- lation was limited to elective supratentorial craniotomies, which limits the generalizability of these results. Whether IV acetaminophen is helpful in managing muscular pain and spasm following posterior fossa procedures, for example, cannot be interpreted from our data. Second, the decision to limit the total daily dosage of acetaminophen in our study to $3 \mathrm{~g}$ may potentially have underestimated the effect of the drug. The decision to limit the maximal dose was an effort to minimize the chance for acetaminophen-related toxicity and comply with the current dosage recommendations of our university pharmacy.

\section{Conclusions}

The addition of IV acetaminophen to treat postoperative craniotomy pain did not result in a significant reduction in narcotic use, as demonstrated in a prospective, double-blinded protocol. Significant differences were not identified in secondary parameters including time to ambulation, time to discharge, episodes of cranial imaging, and episodes of nausea. An improvement in overall pain control was demonstrated. These data provide only modest support for the use of IV acetaminophen to improve postoperative craniotomy pain. Given the importance of opioid minimization while optimizing pain control in postcraniotomy patients, further study of additional adjuncts or combination therapies is needed.

\section{Acknowledgments}

We thank the University of Utah Department of Anesthesia, Investigational Drug Service, Clinical Neurosciences Center Operating Room staff, and Neurocritical Care Unit and Neuroacute Care Unit staff for their logistical support throughout the study. We also thank Kristin Kraus, MSc, for her editorial assistance in the preparation of this manuscript and Vance Mortimer for his audiovisual assistance.

This trial was internally funded by the University of Utah Departments of Neurosurgery and Neurology.

\section{References}

1. Bijur PE, Silver W, Gallagher EJ: Reliability of the visual analog scale for measurement of acute pain. Acad Emerg Med 8:1153-1157, 2001

2. Botting RM: Mechanism of action of acetaminophen: is there a cyclooxygenase 3? Clin Infect Dis 31 (Suppl 5):S202S210, 2000

3. de Gray LC, Matta BF: Acute and chronic pain following craniotomy: a review. Anaesthesia 60:693-704, 2005

4. Hassouneh B, Centofanti JE, Reddy K: Pain management in post-craniotomy patients: a survey of Canadian neurosurgeons. Can J Neurol Sci 38:456-460, 2011

5. Hoefnagel A, Lopez M, Mitchell K, Smith D, Feng C, Nadler $\mathrm{J}$ : Intravenous acetaminophen administration in patients undergoing craniotomy - a retrospective institutional study. J Anesth Clin Res 6:9, 2015

6. Kotak D, Cheserem B, Solth A: A survey of post-craniotomy analgesia in British neurosurgical centres: time for perceptions and prescribing to change? Br J Neurosurg 23:538542, 2009

7. Memis D, Inal MT, Kavalci G, Sezer A, Sut N: Intravenous paracetamol reduced the use of opioids, extubation time, and opioid-related adverse effects after major surgery in intensive care unit. J Crit Care 25:458-462, 2010

8. Molnár C, Simon É, Kazup Á, Gál J, Molnár L, Novák L, 
et al: A single preoperative dose of diclofenac reduces the intensity of acute postcraniotomy headache and decreases analgesic requirements over five postoperative days in adults: a single center, randomized, blinded trial. J Neurol Sci 353:70-73, 2015

9. Nemergut EC, Durieux ME, Missaghi NB, Himmelseher S: Pain management after craniotomy. Best Pract Res Clin Anaesthesiol 21:557-573, 2007

10. Peng K, Jin XH, Liu SL, Ji FH: Effect of intraoperative dexmedetomidine on post-craniotomy pain. Clin Ther 37:11141121, 1121.e1, 2015

11. Raffa RB, Friderichs E, Reimann W, Shank RP, Codd EE, Vaught JL: Opioid and nonopioid components independently contribute to the mechanism of action of tramadol, an 'atypical' opioid analgesic. J Pharmacol Exp Ther 260:275-285, 1992

12. Remy C, Marret E, Bonnet F: Effects of acetaminophen on morphine side-effects and consumption after major surgery: meta-analysis of randomized controlled trials. Br J Anaesth 94:505-513, 2005

13. Roberts G: A review of the efficacy and safety of opioid analgesics post-craniotomy. Nurs Crit Care 9:277-283, 2004

14. Shimony N, Amit U, Minz B, Grossman R, Dany MA, Gonen $\mathrm{L}$, et al: Perioperative pregabalin for reducing pain, analgesic consumption, and anxiety and enhancing sleep quality in elective neurosurgical patients: a prospective, randomized, double-blind, and controlled clinical study. J Neurosurg 125:1513-1522, 2016

15. Sivakumar W, House P, Hoesch R, Martinez J, Duncan N, Ansari S, et al: Intravenous acetaminophen for postoperative supratentorial craniotomy pain-a randomized, double-blinded, placebo-controlled trial. J Neurosurg 122:A1567, 2015 (Abstract)

16. Titsworth WL, Abram J, Guin P, Herman MA, West J, Davis $\mathrm{NW}$, et al: A prospective time-series quality improvement trial of a standardized analgesia protocol to reduce postoperative pain among neurosurgery patients. J Neurosurg 125:1523-1532, 2016
17. Yeh YC, Reddy P: Clinical and economic evidence for intravenous acetaminophen. Pharmacotherapy 32:559-579, 2012

\section{Disclosures}

The authors report no conflict of interest concerning the materials or methods used in this study or the findings specified in this paper.

\section{Author Contributions}

Conception and design: Sivakumar, House, Hoesch. Acquisition of data: Sivakumar, Jensen, Martinez, Duncan. Analysis and interpretation of data: Sivakumar, House, Martinez, Tanana, Riva-Cambrin. Drafting the article: Sivakumar, House. Critically revising the article: Sivakumar, House, Riva-Cambrin. Reviewed submitted version of manuscript: all authors. Approved the final version of the manuscript on behalf of all authors: House. Administrative/technical/material support: House. Study supervision: House.

\section{Supplemental Information \\ Previous Presentations}

An interim analysis was performed after 6 months, the results of which were presented at the 82nd AANS Annual Scientific Meeting, San Francisco, California, April 5-9, 2014.

\section{Current Affiliations}

Dr. Sivakumar: Pacific Neuroscience Institute, John Wayne Cancer Institute, Santa Monica, CA.

\section{Correspondence}

Paul A. House: University of Utah, Salt Lake City, UT. neuropub @hsc.utah.edu. 